\title{
Exosomes: Extracellular communicators of health and disease
}

\author{
Cindy E McKinney* \\ Genetics Department, Edward Via College of Osteopathic Medicine, Spartanburg, SC, USA \\ Stem Cell Lab, Gibbs Research Institute, Spartanburg, SC, USA
}

\begin{abstract}
Exosomes are small microvesicles implicated in cell-cell communication locally and at distant body sites. Evidence suggests that exosomes can modulate normal cell function and are able to elicit disease alterations. For example, exosomes from Alzheimer brains have been shown to carry tau tangles and Parkinson patient exosomes contain $\alpha$-synuclein. These putative "signals of pathology" are derived from intraluminal vesicles formed as endosome products either destined for degradation at the lysosomal-endosomal interface or extruded into extracellular space to be captured by recipient cells. Exosome cargo, packaged from selective cytoplasmic entities, can be proteins, RNAs or other biomolecules like lipids. Exosome cargo from disease cell lines has revealed both translatable mRNA and non-coding RNAs (lncRNA and microRNAs) that potentially have a gene/cell regulatory role. Exosomes appear important for establishing micro-niches for cancer metastasis and are being investigated as participants in neurodegenerative diseases. Numerous exosome proteins and RNAs may be useful as biomarkers of disease. Finally, since exosomes are natural cell shuttles of a variety of cargo and pass the blood-brain barrier, investigators are evaluating whether synthetic cargoes can be loaded into exosomes and used as a therapeutic approach to alleviate disease.
\end{abstract}

\section{Introduction}

Interest in extracellular vesicles (EVs) has exploded in recent years leading to the exploration of exosome content and function. Yet, dissecting the role of EVs and their spectrum of functions remains a complex task. Cells release a variety of extracellular vesicles at their plasma membrane surface (ectosomes (100-1000 nm), apoptotic bodies $(50-5000 \mathrm{~nm})$ and exosomes $(30-140 \mathrm{~nm})$. Exosomes are the smallest of the extracellular vesicle types and are released from non-apoptotic cells [1]. Pan and Johnstone [2] first observed exosomes in reticulocytes and hypothesized that they were a way for that cell to extrude extra cell membrane and/or act as carriers of waste products expelled from the cells. Despite the explosion of research into the role of exosomes found in all organisms, we are still learning about their biogenesis, trafficking to the extracellular space and their physiological roles in health and disease. This short review summarizes the current focus in exosome research.

\section{Biogenesis and trafficking}

Exosomes are the smallest of the extracellular vesicle types. They form by invagination of endosome membrane into the endosome space capturing cytoplasmic content generating intraluminal vesicles (ILVs). Endosomes loaded with ILVs become a multivesicular body (MVB) (Figure 1). Exosomes [3] are membrane bound vesicles approximately $30-140 \mathrm{~nm}$ in size and within the vesicle contain a variety of cargo. Exosomes are extruded into the extracellular space and can enter recipient cells through (1) endocytosis at the recipient cell's plasma membrane surface, (2) recipient cell receptor-mediated endocytosis and/or (3) direct fusion with the recipient cell's plasma membrane [4] or (5) they can enter the organism's circulation from the extracellular space. An alternate fate of the ILV containing MVB is fusion with the lysosome where the MVB contents are degraded. Assembly of exosomes occurs in at least three ways (1) by protein sorting of specific ubiquinated proteins and the ESCRT (Endosomal Sorting Complex Required for Transport) machinery [5], (2) by association with lipid rafts contained in the exosomes lipid bilayer membrane [6] and (3) by a ceramide dependent pathway that captures biomolecules [7]. Specific proteins are required to load the exosome (ESCRT complex), energy from ATP lysis (Rab GTPases) is needed for release at the plasma membrane and proteins embedded in the exosome lipid membrane are needed for recognition and capture at the recipient cell's surface (transpanins, flotillins and others). The ESCRT complex and associated proteins are used to sort and cluster ubiquinated proteins like receptors [8]. The tetraspanins along with CD9 and CD63 initiate exosome formation by clustering in microdomain regions of the endosome membrane. Segregation of proteins in the microdomains occurs and an additional recruitment of ESCRT complex proteins begins invagination of the endosomal membrane capturing cytosolic cargo in the ILVs (reviewed by Kalra [8]). Also, It is shown that there is an ESCRT complex independent pathway for formation of ILVs; this glycoprotein pathway involves the formation of lipid rafts [9] where ceramide may be a key component. Oligodendrocytes seem to form exosomes via sphingomyleinase cleavage of sphingomyelin to ceramide [7]. Ceramide induces invagination and then formation of ILVs. Subsequent steps in biogenesis involve moving the MVB to the plasma membrane surface, fusion with the plasma membrane and release of the exosomes. YKY6 SNARE protein and Rab GTPases direct exosomes to the plasma membrane surface $[4,10]$. Interestingly,

*Correspondence to: Cindy E McKinney, Genetics Department, Edward Via College of Osteopathic Medicine, Spartanburg, SC, USA; E-mail: david.stejskal@smn.agel.cz

Received: August 10, 2016; Accepted: August 19, 2016; Published: August 23, 2016 


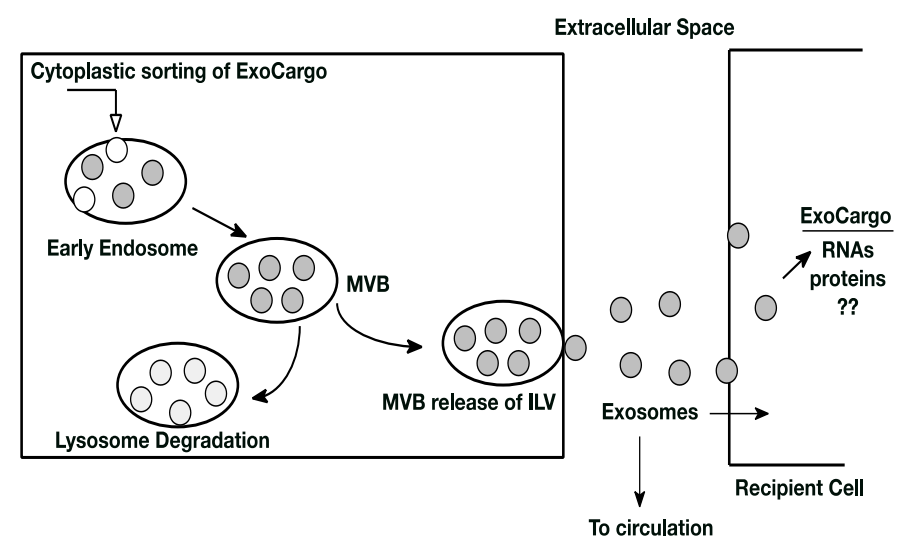

Figure 1. Generation and trafficking of exosomes: Exosomes are formed in the early exosome as intraluminal vesicles (ILV) from selectively sorted cytoplasmic contents. These then become multivesicular bodies (MVB). MVB have two fates; they can fuse with lysosomes where their contents are degraded or they can fuse with the cytoplasmic membrane and release their ILVs (now exosomes) into the extracellular space. Nearby recipient cells can locally capture the exocargo that may modulate it's functions. Exosomes from the extracellular space can also enter the circulation and be captured at distant cell sites where, again, they may modulate that cells function. Exosomes from any cell source (liver, brain) are captured by any other cell (heart, pancreas) thereby providing a unique cell-cell communication pathway.

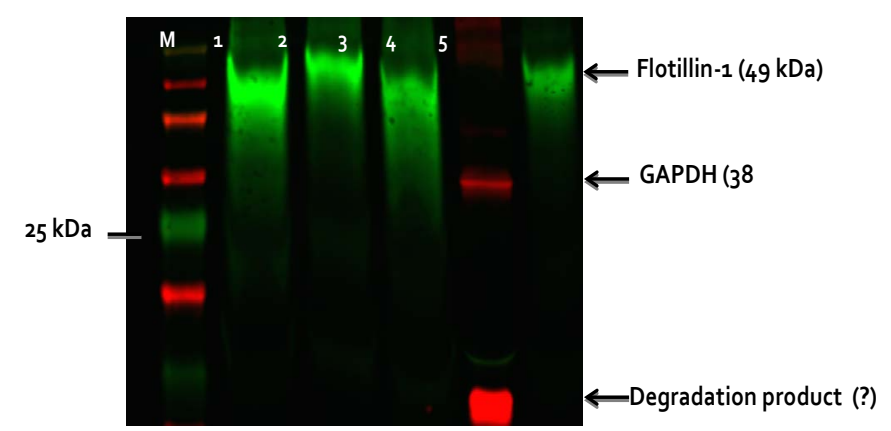

Figure 2. Western blot of exosomes isolated from neural Type 2 Gaucher lines and cell lysates.

Exosomes were isolated from conditioned culture medium with ExoQuick (SBI). Lane 1: exosomes from patient 1260 early neurons, Lane 2: exosomes from VCOM 107 control neurons, Lane 3: exosomes from patient 1260 late neurons, Lane 4: BJ control neuron cell lysate and Lane 5: exosomes from BJ control neurons. Western was run with $10 \%$ SDS-PAGE gel (25 ug protein/lane), blotted to PVDF and probed with indicated primary antibodies for flotillin-1 found in exosomes and GPADH a housekeeping enzyme. Although GAPDH has been reported in some exosomes (see Exocarta.org) it was not seen in the exosomes isolated from these Gaucher neurons differentiated from our iPSC lines made from patient fibroblasts. " $\mathrm{M}$ " is a fluorescent marker lane.

the exosome membrane composition is not the same as that of the cell membrane [8]. There are two fates for ILVs contained in a MVB (1) they can fuse with a lysosome directing their cargo for degradation or (2) they can be released at the cell's plasma membrane into the extracellular space [8]. It is these ILVs that are termed exosomes. The cellular process that designates ILV containing MVBs to either the degradation or extracellular pathways remains elusive.

\section{Exosome cargo}

Exosomes are lipid bilayer membrane bound packages of cytosolic cargo derived in the endosome. The molecular cargo contained in exosomes is reported to include protein, RNAs, lipids, metabolites and, although still debated, DNA. These are captured cytosolic molecules loaded into the exosomes and released to the extracellular space [11]. However, comparisons of the exosome cargo and the cytosolic content from which it is derived are often different suggesting a selective sorting mechanism used to load the ILV destined to be an exosome. Due to their method of formation, exosomes contain common proteins derived from cytosolic components. Because of their endosomal origins, exosome composition includes cellular biomarkers (ExoCarta). Those biomarker proteins commonly reported and identified with exosomes are: GTPases, flotillin-1, annexin, heat shock proteins (CD9, CD63, CD81) and other proteins associated with multivesiclular body biogenesis (e.g., Alix and TSG101) as well as many others $[3,12]$ (Figure 2). Exosomes are also found to be enriched in lipids including, ceramide, cholesterol, and sphingolipids and have a surface signature of glycans and polysaccharides $[8,13]$. Exosomes are released into the extracellular space and can circulate in the blood and cerebrospinal fluid, be received at a recipient cell's interface where they are internalized and can influence recipient cell functioning [14] or excreted in urine. DNA Cargo: DNA from exosome like vesicles was spontaneously emitted from mouse brain tumor cells and contained chromatin coated H-Ras DNA [15]. The authors showed that the $\mathrm{H}$-Ras DNA was able to induce cell proliferation in non-transformed cells. While other groups have identified DNA in exosome vesicles transcription to functional RNAs is debated. This is another enigma to be resolved as exosome research progresses.

\section{RNA cargo}

Exosomes from many cell types carry RNAs (messenger RNA (mRNA), microRNA (miRNA) and long non-coding RNA (lncRNA) that seem to alter cellular activity in the recipient cell [16]. Valadi et al. [17] reported the presence of RNA species in exosomes and also showed that this RNA is functional in that it could be translated into proteins. It appears that RNAs, captured at the endosome interface from the cell cytoplasm, then have two fates (1) mRNA translation into protein or (2) miRNA repression of translation $[16,18,19]$. Thus, exosome RNA (exoRNA) can be a powerful tool for coordinating cell functions within an organism. Since exoRNA profiles are distinct from those in the donor cell's cytoplasm, there appears to be selective packaging of this cargo but the process remains unknown. Microarray screens have shown a portion of the exoRNA content is miRNA. Eckstom et al. [16] reported that some miRNAs are enriched and some decreased when compared to cell cytoplasm RNA content, this again argues for selective packaging to the exosome pathway.

\section{Protein cargo}

Proteins destined for different cellular compartments frequently contain a 5' signal sequence to direct them to the appropriate cellular compartment (e.g., a nuclear localization signal (NLS) but it remains undetermined how the proteins found in exosomes are selected for vesicle encapsulation. It is noted that ubiquination [20] of proteins may direct them to the ILVs and ESCRT complex proteins do recognize ubiquinated proteins. Tetraspanin rich (CD9, CD63, CD81 and CD82) lipid raft domains also may recruit proteins to the ILVs [20]. Exosomal proteins are representative of the protein mix in the source cell, for this reason, many proteins may be employed as cellular biomarkers of pathology. It is reported that intracellular proteins from the endoplasmic reticulum, mitochondria and nucleus are under-represented in exosomes [4,21,22]. Exosomes are enriched in proteins such as ALIX, TSG101 and a variety of heat shock proteins (CD9, CD63 and CD81). Weng et al. [23] reported that an analysis of exosome proteins obtained from HeLa cell tissue culture supernatant encompassed 6299 protein groups from 5120 genes and that $97 \%$ of them were reported in ExoCarta. Some of these proteins were identified as protein degradation intermediates. 
There are two useful databases (ExoCarta: http://www.exocarta.org and Vesiclepedi: microvesicles.org [24]) that contain information across organisms and cells on exosome cargo isolated from different cell types with links to other information on exosomes and microvesicles found in cells to date. The exoRNA portal (exrna.org) has information that summarizes data on extracellular RNA and provides links to additional information.

\section{Isolation and characterization methods}

Exosome vesicles (30-140 nm) derived from endosome pathways [25] are membrane bound packets of proteins and various classes of RNAs that appear to be unique for each cell type or cell line [3]. Exosome content characteristically reflects the physiological state of the cells that secrete them, for example, exosomes from Alzheimer's brains typically contain aggregates of tau protein [26]. They are isolated from many biological fluids including urine and blood and from tissue culture medium. It is estimated that blood typically has an exosome load of $5-50 \mu \mathrm{g} / \mathrm{ml}$. The circulation captures all the exosomes secreted from different cell types so selection of subpopulations of, for example, kidney exosomes from blood becomes problematic. Initially, exosomes were isolated by selective centrifugation steps that removed cellular debris and then a final ultracentrifugation step (with or without a sucrose gradient) that captured exosomes (110,000 x g for 90 minutes) $[25,27]$. The ultracentrifugation protocol isolates vesicles of $<100 \mathrm{~nm}$ and this size distribution may also contain small apoptotic bodies [3]. One disadvantage of isolating exosomes by ultracentrifugation is the shear forces placed on the vesicles over long centrifugation times [28] may limit the recovery of exosomes. Thus, captured exosomal cargo is less using this method [1]. However, many in the field still consider ultracentrifugation the "gold standard" for acquiring exosomes. Isolating exosomes from cell culture medium requires that any FBS in the medium be exosome-free (commercially available) as this medium supplement contains bovine exosomes that may interfere with downstream analyses [21] and reviewed in [3]. Other methods of isolating and collecting exosomes are immune-affinity capture using an antibody to protein(s) on the surface of the exosome linked to beads [29]. The exosomes are bound by isolation medium and they may not be able to be released and utilized in downstream applications. Size exclusion chromatography coupled with centrifugation steps to remove cellular debris can also isolate selectively sized exosomes $[28,30]$. Finally, a method based on addition of a polyethylene glycol (PEG) substrate uses low speed centrifugation to precipitate exosomes after an overnight incubation at $4^{\circ} \mathrm{C}[31,32]$. This method is reported to yield high quality exosomes and is available as a commercial reagent (ExoQuick). However, it is not clear if PEG remaining in the exosome preparation after centrifugation interferes with downstream applications. Post-isolation methods, such as capturing PEG on a G-25 column could address this issue [28]. At present most groups investigating exosome activity select one isolation method and work within the confines of that procedure. Thus, comparison of results between methods should be carefully evaluated. Whatever isolation method is employed, it requires validation to confirm that nonexosomal material is not contaminating the preparation leading to errors in analyses. Second, the exosome isolation method should maintain the integrity of the vesicle and its content. This is frequently analyzed by EM or by using Nanosight instrumentation.

\section{Exosome function}

Extracellular vesicles including exosomes act as transport vehicles. The vesicles are another method cells can utilize to communicate both short and long range signaling information [8]. The exosomes cargo, carrying the effector signals, is representative of both the exosomes cell origin and the physiological state of the originating cell (healthy or diseased) [33]. After screening exosome RNA content from the HMC1 cell line, Eckstrom et al. [16] reported the identity of the pathways the RNAs were located to. The networks with the highest score were cellular and hematological development, protein synthesis, modification and folding, RNA post-transcriptional modifications and cellular assembly. The top five previously established pathways enumerated were: (1) oxidative phosphorylation, (2) mitochondrial dysfunction, (3) EIF2 signaling, (4) regulation of actin-based mobility by Rho and (5) protein ubiquination pathways. This suggests a diversity of function for the exosomes from these studies [16].

Exosomes are acquired at any recipient cell surface; for example, neural cells can acquire liver cell exosomes. This generalized ability to capture exosomes from surrounding cell exudate or blood circulation increases the complexity of the communication pathways in the body [34]. Other roles documented for exosomes include morphogen signaling in development [20] and immunological mediators [20]. Finally, exosomal miRNAs and lncRNAs may function in, as yet, unidentified regulatory roles.

\section{Roles in neurodegenerative disease}

Exosomes are released by all cell types examined in the CNS either to the intracellular space or into the cerebral spinal fluid (CSF) or blood circulation $[4,35]$. Exosomes may act both positively and negatively on recipient cells. As described, exosomes can remove cellular waste, communicate signaling information between local and distant sites and may be vehicles to transfer pathogens like infectious viral particles [36] or prions [37]. Thus, exosomes are cellular communicators of disease and they can be captured from CSF and medium from cultured neural cells. They have been studied in several neurodegenerative diseases including Alzheimer's disease [38,39], Creutzfeldt-Jakob Syndrome [40,41], Parkinson's [42,43] and Amyotrophic Lateral Sclerosis (ALS) [44]. Extracellular vesicles from one neural cell type may interact with other neurons, astrocytes, microglia, and oligodendrocytes transporting both healthy and diseased proteins, lipids or RNA species [4]. In Alzheimer's disease the accumulation of amyloid plaques, insoluble protein complexes, have been isolated as content in exosomes [45]. Transfer of the amyloid complexes via exosomes to other neural cells may "seed" protein misaggregation in the recipient cells and, thus, spread disease [46]. This mode of exosome transfer of aggregated protein is also reported for Parkinson's disease where a-synculein is found in exosomes and ALS where mutant SOD1 is located in glia-derived exosomes [4]. What remains to be investigated is if the exosomes are simply a transport vehicle for disease spread or if their composition is also contributing to disease. Recent investigative reports do suggest a role for mutant proteins encased by exosomes playing a role in transmitting neurological disease. For example, a paper investigating the progressive nature of ALS, found mutations in the FUS (Fused in Sarcoma) gene in both sporadic and familial ALS [47]. Protein pull-down analyses identified the proteins associated with the normal and mutant FUS gene product. The authors showed these proteins are active in many cellular pathways including nuclear organization, transcription, RNA transport and stress response, thus, demonstrating a broad role for FUS in cellular activity. FUS protein and many of it's interacting proteins were found localized to exosomes suggesting that release of these exosomes to adjacent recipient cells is one route of disease spread in these ALS patients. 
Frontotemporal lobular degeneration (FTLD) primarily affects patients younger than 65 years where loss of function is in the frontal and anterior temporal brain lobes [48]. Upwards of 50\% of patients have a family history and genetic factors are considered the primary cause of FTLD. Granulin mutations (GRN) are associated with FTLD that cause haploinsufficiency or loss of progranulin protein in these patients. Benussi and colleagues [48] cultured fibroblasts from 16 FTLD patients with GRN mutations and appropriate cell controls. They found progranulin associated with exosomes from culture medium in its N-glycosylated form and the quantity of GRN was reduced in exosomes from the FTLD fibroblast cultures. They also show that exosome numbers from FTLD patients, where GRN is deficient, are reduced and contain LAMP1, a lysosomal protein. These findings were not consistent with cell apoptosis or impaired cell viability. Progranulin is secreted as a soluble neurotrophic factor that promotes neurite outgrowth and neuron viability but is also found in exosomes. Thus, loss of progranulin in FTLD may impact development of neurodegeneration by a reduction of exosome extracellular communication.

Exosome release in some neurons is coupled to glutamate receptor activation as $\mathrm{Ca}^{+2}$ enters' the neuron via $\mathrm{N}$-methyl-D-aspartate (NMDA) receptors [49]. Exosomes released from cultured N2a cells were captured by both neurons and glial cells. In contrast, GFPTTC (green fluorescent protein coupled to non-toxic tetanus toxin C-fragment) labeled exosomes released by synaptic activation were selectively transferred only to other neurons at their synapses [35]. This work demonstrates a specific signal for release of exosomes from neurons and that these exosomes are selectively accrued by other neural cells. This phenomenon apparently occurs by receptor recognition at the recipient cell interface or recipient cell endocytosis via the plasma membrane [39]. Exosome cargo may affect neural cell function in positive ways mediating oxidative stress [49] and altering gene expression patterns in recipient neurons [50].

Exosome shuttle RNAs (esRNA) are found in exosomes and contain small RNAs and miRNA that can enter the extracellular space and affect cellular function in recipient cells [46]. Many observations [45] support the conclusion that exosomes from diseased cells transfer both coding RNA and non-coding miRNA that may alter cell function producing neurotoxicity. Neurons communicate with their support cells (astrocytes and neuroglia) responding to peripheral stimuli. It is not hard to envision extracellular transport of exosomes as a signaling method both for healthy and disease outcomes. Whatever the source of RNAs or protein found in exosomes and the relative ease of capture from urine, CSF and blood, there is increasing interest in using exosome preparations as potential biomarkers of disease [4].

Exosomes may act as mediators of neurological cancers. Glioblastomas have been characterized to release a variety of extracellular vesicles (exosomes and the larger oncosomes) whose composition diverges from cytoplasmic content [51-53]. These "tumor vesicles" are reported to increase a variety of oncogenic processes that promote tumor growth and development, like immune suppression, establishment of tumor micro-niches and angiogenesis [52,54]. Li et al. [55] showed that glioblastoma extracellular vesicles carry both noncoding RNAs and RNAs to recipient cells that alter transcriptional profiles. Extensive analysis of neural tumor cell's exosome cargo is needed to establish what is being transferred via this route of extracellular communication and how it is abnormally altering the function of the receiving cell. The role of exosomes from tumor cells found in all parts of the body is reviewed in [3,56-58].

\section{Potential therapeutic roles for exosomes}

\section{Diagnostic or prognostic biomarkers}

Exosomes are suggested as promising carriers of biomarkers where protein cargo profiling may be especially informative [59] and where exosomes could easily be captured from the patient's blood. Exosomes are accessible from the blood, urine, CSF and other body secretions making collection methods low risk and minimally invasive. They might be particularly useful in capturing information from different tumor types, for prognostic staging of tumor progression and, potentially, for identifying a patient specific drug regimen [60]. The role of exosomes as biomarkers in cancer and its metastasis is reviewed in [61]. Another application using blood exosomes from neonates to assess neurotoxicity is profiled in Gillet et al. [62]. Profiling exosome miRNA to identify biomarker candidates for early Alzheimer's disease is discussed in Kumar and Reddy [63]. Cheow et al. [64] showed the utility of using exosomes as biomarkers for therapy of myocardial ischemic injury. In a neurodegenerative disease example, mutations in LRRK2 (leucine rich repeat kinase 2) are one of the most frequent causes of familial Parkinson's disease and suspected in idiopathic Parkinson's disease. The mutation G2019S in LRRK2 allows phosphorylation of the serine in the now mutant protein and this could act as a potential biomarker of Parkinson's disease. Fraser et al. [65,66] captured urine exosomes from Parkinson's patients that contain LRRK2 and showed that the mutant LRRK2 serine was indeed phosphorylated. The level of phosphorylated serine in the exosome was significant when compared to control patients. The phosphorylated serine level also was found to correlate to the degree of cognitive dysfunction seen in idiopathic Parkinson's patients [66]. The potential of exosomes to be used as biomarker appears limitless but takes extensive validation to arrive at a potent biomarker [67]. As these analyses broaden and become more sophisticated and reliable, exosome biomarkers will enter the biomedical mainstream and likely be approachable as liquid biopsies [68].

\section{Exosome carriers of ex vivo therapy}

Exosomes have four characteristics that make them a putative therapy approach for treating both metabolic disease and various forms of cancer. First, they are a naturally encapsulated package that is able to avoid cellular degradation [4]. Exosomes are received at all cellular membranes, carried in the blood or CSF and able to cross the bloodbrain barrier making them a good extracellular candidate as a carrier of therapeutics. Exosomes might also be better tolerated by the immune system since they are natural nanocariers of cellular cargo [69,70]. Lastly, exosome cargo can be substituted for synthetic proteins or drugs that could induce therapeutic reactions. As an example, bone regeneration has focused on stimulating repair but has not investigated the putative cell-cell communication need for stimulation and continuity of repair. Exosomes exhibit both stimulation of repair in osteogenic cells and can carry "messages" between cells [71]. Hence, it appears that exosomes can directly regulate bone repair by stimulating osteoblast function. Studies of the exosomes role in alleviating pathophysiology require much more investigation. It is proposed that exosomes, loaded with specialized cargoes, could also be engineered by adding receptor ligands to target specific cell populations. This approach offers exciting opportunities for treatment of cancer, neurodegenerative or metabolic disease. Reproducible exosome therapies await assessment of delivery to selected tissues and validation of reaching a therapeutic threshold of efficacy. These developments were recently reviewed in [67,72-75]. 


\section{Conclusions}

A better understanding of the molecular mechanisms that regulate formation of ILVs into MVBs, selection of ILV cargoes and trafficking of MVBs to either lysosomes for degradation or to the plasma membrane for release will advance our understanding of diseases where pathogenic proteins, lipids or infectious agents are present in the exosome [76]. Critical to understanding the role exosomes play in health and disease is defining the contents of exosomes and understanding how exosomes from diseased cells differ from healthy cells. Exosomes from diseased cells should be a rich source of identifiable biomarkers for a specific pathology. Due to the nature of the exosome, a bilayer lipid bound vesicle that protects its contents from proteolysis, the exosome itself, in future, may be loaded with synthetic and therapeutic cargoes to address amelioration of cancer, neurodegenerative and metabolic diseases. Many avenues of exosome research have progressed over the past 10 years and one anticipates new insights and broader knowledge of the exosomes role in altering a cell's functionality and the potential use of exosomes as biomarkers for pathology and for treatment of disease.

\section{References}

1. Livshits MA, Khomyakova E, Evtushenko EG, Lazarev VN, et al. (2015) Isolation of exosomes by differential centrifugation: Theoretical analysis of a commonly used protocol. Sci Rep 5: 17319. [Crossref]

2. Pan BT, Johnstone RM (1983) Fate of the transferrin receptor during maturation of sheep reticulocytes in vitro: selective externalization of the receptor. Cell 33: 967-978. [Crossref]

3. Szatanek R, Baran J, Siedlar M, Baj-Krzyworzeka M (2015) Isolation of extracellular vesicles: Determining the correct approach (Review). Int $J$ Mol Med 36: 11-17. [Crossref]

4. Basso M, Bonetto V (2016) Extracellular Vesicles and a Novel Form of Communication in the Brain. Front Neurosci 10: 127. [Crossref]

5. Hurley JH (2015) ESCRTs are everywhere. EMBO J 34: 2398-2407. [Crossref]

6. Janas T, Janas MM, Sapo $\AA$, K, Janas T (2015) Mechanisms of RNA loading into exosomes. FEBS Lett 589: 1391-1398. [Crossref]

7. Trajkovic K, Hsu C, Chiantia S, Rajendran L, Wenzel D, et al. (2008) Ceramide triggers budding of exosome vesicles into multivesicular endosomes. Science 319: 1244-1247. [Crossref]

8. Kalra H, Drummen GP, Mathivanan S (2016) Focus on Extracellular Vesicles: Introducing the Next Small Big Thing. Int J Mol Sci 17: 170. [Crossref]

9. Phuyal S, Skotland T, Hessvik NP, Simolin H, et al. (2015) The ether lipid precursor hexadecylglycerol stimulates the release and changes the composition of exosomes derived from PC-3 cells. $J$ Biol Chem 290: 4225-4237.

10. Colombo M, Raposo G, Thery C (2014) Biogenesis, secretion, and intercellular interactions of exosomes and other extracellular vesicles. Annu Rev Cell Dev Biol 30 : 255-289. [Crossref]

11. Villarroya-Beltri C, Baixauli F, Gutiérrez-Vázquez C, Sánchez-Madrid F, Mittelbrunn M (2014) Sorting it out: regulation of exosome loading. Semin Cancer Biol 28: 3-13. [Crossref]

12. Penfornis P, Vallabhaneni KC, Whitt J, Pochampally R (2016) Extracellular vesicles as carriers of microRNA, proteins and lipids in tumor microenvironment. Int $J$ Cancer 138: 14-21. [Crossref]

13. Zaborowski MP, Balaj L, Breakefield XO, Lai CP (2015) Extracellular Vesicles: Composition, Biological Relevance, and Methods of Study. Bioscience 65: 783-797.

14. Yáñez-Mó M, Siljander PR3, Andreu Z, et al. (2015) Biological properties of extracellular vesicles and their physiological functions. J Extracell Vesicles 4: 27066. [Crossref]

15. Lee TH, Chennakrishnaiah S, Audemard E, Montermini L, Meehan B, et al. (2014) Oncogenic ras-driven cancer cell vesiculation leads to emission of double-stranded DNA capable of interacting with target cells. Biochem Biophys Res Commun 451: 295301. [Crossref]

16. Ekström K, Valadi H, Sjöstrand M, Malmhäll C, Bossios A, et al. (2012) Characterization of mRNA and microRNA in human mast cell-derived exosomes and their transfer to other mast cells and blood CD34 progenitor cells. J Extracell Vesicles 1. [Crossref]

17. Valadi H, Ekström K, Bossios A, Sjöstrand M, Lee JJ, et al. (2007) Exosome-mediated transfer of mRNAs and microRNAs is a novel mechanism of genetic exchange between cells. Nat Cell Biol 9: 654-659. [Crossref]

18. Bryniarski K, Ptak W, Martin E, Nazimek K, Szczepanik M, et al. (2015) Free Extracellular miRNA Functionally Targets Cells by Transfecting Exosomes from Their Companion Cells. PLoS One 10: e0122991. [Crossref]

19. Momen-Heravi F, Bala S, Bukong T, Szabo G (2014) Exosome-mediated delivery of functionally active miRNA-155 inhibitor to macrophages. Nanomedicine 10: 15171527. [Crossref]

20. Keller S, Sanderson MP, Stoeck A, Altevogt P (2006) Exosomes: from biogenesis and secretion to biological function. Immunol Lett 107: 102-108. [Crossref]

21. Lötvall J, Hill AF, Hochberg F, Buzás EI, Di Vizio D, et al. (2014) Minimal experimental requirements for definition of extracellular vesicles and their functions: a position statement from the International Society for Extracellular Vesicles. $J$ Extracell Vesicles 3: 26913. [Crossref]

22. Keerthikumar S, Gangoda L, Liem M, Fonseka P, Atukorala I, et al. (2015) Proteogenomic analysis reveals exosomes are more oncogenic than ectosomes. Oncotarget 6: 15375-15396. [Crossref]

23. Weng Y, Sui Z, Shan Y, Jiang H, et al. (2016) In-Depth Proteomic Quantification of Cell Secretome in Serum-Containing Conditioned Medium. Anal Chem 88: 4971-4978. [Crossref]

24. Kalra H, Simpson RJ, Ji H, Aikawa E, Altevogt P, et al. (2012) Vesiclepedia: compendium for extracellular vesicles with continuous community annotation. PLoS Biol 10: e1001450. [Crossref]

25. Valencia K, Lecanda F, (2016) Microvesicles: Isolation, Characterization for In Vitro and In Vivo Procedures. Methods Mol Biol 1372: 181-192. [Crossref]

26. Malm T, Loppi S, Kanninen KM (2016) Exosomes in Alzheimer's disease. Neurochem Int 97: 193-199. [Crossref]

27. Raposo G, Nijman HW, Stoorvogel W, Liejendekker R, Harding CV, et al. (1996) B lymphocytes secrete antigen-presenting vesicles. J Exp Med 183: 1161-1172. [Crossref]

28. Taylor DD, Shah S (2015) Methods of isolating extracellular vesicles impact downstream analyses of their cargoes. Methods 87: 3-10. [Crossref]

29. Greening DW, Xu R, Ji H, Tauro BJ, Simpson RJ, et al. (2015) A protocol for exosome isolation and characterization: evaluation of ultracentrifugation, density-gradien separation, and immunoaffinity capture methods. Methods Mol Biol 1295: 179-209.

30. Witwer KW, Buzás EI, Bemis LT, Bora A, Lässer C, et al. (2013) Standardization of sample collection, isolation and analysis methods in extracellular vesicle research. $J$ Extracell Vesicles 2013: 2. [Crossref]

31. Taylor DD (2015) Isolation and molecular characterization of extracellular vesicles Methods 87: 1-2. [Crossref]

32. Weng Y, Sui Z, Shan Y, Hu Y, Chen Y, et al. (2016) Effective isolation of exosomes with polyethylene glycol from cell culture supernatant for in-depth proteome profiling. Analyst 141: 4640-4646. [Crossref]

33. Lo Cicero A, Stahl PD, Raposo G (2015) Extracellular vesicles shuffling intercellular messages: for good or for bad. Curr Opin Cell Biol 35: 69-77. [Crossref]

34. Hannafon BN, Ding WQ (2013) Intercellular communication by exosome-derived microRNAs in cancer. Int J Mol Sci 14: 14240-14269. [Crossref]

35. Chivet M, Javalet C, Laulagnier K, Blot B, Hemming FJ, et al. (2014) Exosomes secreted by cortical neurons upon glutamatergic synapse activation specifically interact with neurons. J Extracell Vesicles 3: 24722. [Crossref]

36. Sampey GC, Meyering SS, Asad Zadeh M, Saifuddin M, Hakami RM, et al. (2014) Exosomes and their role in CNS viral infections. J Neurovirol 20: 199-208. [Crossref]

37. Fevrier B, Vilette D, Archer F, Loew D, Faigle W, et al. (2004) Cells release prions in association with exosomes. Proc Natl Acad Sci U S A 101: 9683-9688. [Crossref]

38. Medina M, Avila J (2014) The role of extracellular Tau in the spreading of neurofibrillary pathology. Front Cell Neurosci 8: 113. [Crossref]

39. Rajendran L, Bali J, Barr MM, Court FA, Krämer-Albers EM5, et al. (2014) Emerging roles of extracellular vesicles in the nervous system. J Neurosci 34: 15482-15489. [Crossref]

40. Vella LJ, Hill AF (2008) Generation of cell lines propagating infectious prions and 
the isolation and characterization of cell-derived exosomes. Methods Mol Biol 459: 69-82. [Crossref]

41. Vella LJ, Sharples RA, Nisbet RM, Cappai R, Hill AF (2008) The role of exosomes in the processing of proteins associated with neurodegenerative diseases. Eur Biophys $J$ 37: 323-332. [Crossref]

42. Shi M, Kovac A, Korff A, Cook TJ, Ginghina C, et al. (2016) CNS tau efflux via exosomes is likely increased in Parkinson's disease but not in Alzheimer's disease. Alzheimers Dement . [Crossref]

43. Tomlinson PR, Zheng Y, Fischer R, Heidasch R, Gardiner C, et al. (2015) Identification of distinct circulating exosomes in Parkinson's disease. Ann Clin Transl Neurol 2: 353361. [Crossref]

44. Basso M, Pozzi S, Tortarolo M, Fiordaliso F, Bisighini C, et al. (2013) Mutant copperzinc superoxide dismutase (SOD1) induces protein secretion pathway alterations and exosome release in astrocytes: implications for disease spreading and motor neuron pathology in amyotrophic lateral sclerosis. J Biol Chem 288: 15699-15711. [Crossref]

45. Vella LJ, Hill AF, Cheng L (2016) Focus on Extracellular Vesicles: Exosomes and Their Role in Protein Trafficking and Biomarker Potential in Alzheimer's and Parkinson's Disease. Int J Mol Sci 17: 173. [Crossref]

46. Bellingham SA, Guo BB, Coleman BM, Hill AF (2012) Exosomes: vehicles for the transfer of toxic proteins associated with neurodegenerative diseases? Front Physiol 3: 124. [Crossref]

47. Kamelgarn M, Chen J, Kuang L, Arenas A, Zhai J5, et al. (2016) Proteomic analysis of FUS interacting proteins provides insights into FUS function and its role in ALS. Biochim Biophys Acta 1862: 2004-2014. [Crossref]

48. Benussi L, Ciani M, Tonoli E, Morbin M, Palamara L, et al. (2016) Loss of exosomes in progranulin-associated frontotemporal dementia. Neurobiol Aging 40: 41-49. [Crossref]

49. Frühbeis C, Fröhlich D, Kuo WP, Amphornrat J, Thilemann S, et al. (2013) Neurotransmitter-triggered transfer of exosomes mediates oligodendrocyte-neuron communication. PLoS Biol 11: e1001604. [Crossref]

50. Fröhlich D, Kuo WP, Frühbeis C, Sun JJ, Zehendner CM, et al. (2014) Multifaceted effects of oligodendroglial exosomes on neurons: impact on neuronal firing rate, signal transduction and gene regulation. Philos Trans R Soc Lond B Biol Sci 369: 1652.

51. Skog J, Wurdinger T, van Rijn S, Meijer D, Gainche L, et al. (2008) Glioblastoma microvesicles transport RNA and proteins that promote tumour growth and provide diagnostic biomarkers. Nat Cell Biol 10: 1470-1476. [Crossref]

52. D'Asti E, Garnier D, Lee TH, Montermini L, Meehan B, et al. (2012) Oncogenic extracellular vesicles in brain tumor progression. Front Physiol 3: 294. [Crossref]

53. Garnier D, Magnus N, Meehan B, Kislinger T, Rak J, et al. (2013) Qualitative changes in the proteome of extracellular vesicles accompanying cancer cell transition to mesenchymal state. Exp Cell Res 319: 2747-2757. [Crossref]

54. Vader P, Breakefield XO, Wood MJ (2014) Extracellular vesicles: emerging targets for cancer therapy. Trends Mol Med 20: 385-393. [Crossref]

55. Li CC, Eaton SA, Young PE, Lee M, Shuttleworth R, et al. (2013) Glioma microvesicles carry selectively packaged coding and non-coding RNAs which alter gene expression in recipient cells. RNA Biol 10: 1333-1344. [Crossref]

56. Lopatina T, Gai C, Deregibus MC, Kholia S, Camussi G, et al. (2016) Cross Talk between Cancer and Mesenchymal Stem Cells through Extracellular Vesicles Carrying Nucleic Acids. Front Oncol 6: 125. [Crossref]

57. Desrochers LM, Bordeleau F, Reinhart-King CA, Cerione RA, et al. (2016)
Microvesicles provide a mechanism for intercellular communication by embryonic stem cells during embryo implantation. Nat Commun 7: 11958. [Crossref]

58. Suchorska WM, Lach MS (2016) The role of exosomes in tumor progression and metastasis (Review). Oncol Rep 35: 1237-1244. [Crossref]

59. Sandfeld-Paulsen B, Jakobsen KR, Bæk R, Folkersen BH, Rasmussen TR, et al (2016) Exosomal Proteins as Diagnostic Biomarkers in Lung Cancer. J Thorac Oncol. [Crossref]

60. Min L, Shen J, Tu C, Hornicek F, et al. (2016) The roles and implications of exosomes in sarcoma. Cancer Metastasis Rev. [Crossref]

61. Soung YH, Nguyen T, Cao H, Lee J, Chung J (2016) Emerging roles of exosomes in cancer invasion and metastasis. BMB Rep 49: 18-25. [Crossref]

62. Gillet V, Hunting DJ, Takser L (2016) Turing Revisited: Decoding the microRNA Messages in Brain Extracellular Vesicles for Early Detection of Neurodevelopmental Disorders. Curr Environ Health Rep 3: 188-201. [Crossref]

63. Kumar S, Reddy PH (2016) Are circulating microRNAs peripheral biomarkers for Alzheimer's disease? Biochim Biophys Acta 1862: 1617-1627. [Crossref]

64. Cheow ES, Cheng WC, Lee CN, de Kleijn D, Sorokin V, et al. (2016) Plasma-derived extracellular vesicles contain predictive biomarkers and potential therapeutic targets for myocardial ischemic injury. Mol Cell Proteomics 15: 2628-2640. [Crossref]

65. Fraser KB, Moehle MS, Alcalay RN, West AB2; LRRK2 Cohort Consortium (2016) Urinary LRRK2 phosphorylation predicts parkinsonian phenotypes in G2019S LRRK2 carriers. Neurology 86: 994-999. [Crossref]

66. Fraser KB, Rawlins AB, Clark RG, Alcalay RN, Standaert DG, et al. (2016) Ser(P)-1292 LRRK2 in urinary exosomes is elevated in idiopathic Parkinson's disease. Mov Disord. [Crossref]

67. Stremersch S, De Smedt SC, Raemdonck K (2016) Therapeutic and diagnostic applications of extracellular vesicles. J Control Release. [Crossref]

68. He M, Zeng Y (2016) Microfluidic Exosome Analysis toward Liquid Biopsy for Cancer. J Lab Autom 21: 599-608. [Crossref]

69. Li X, Tsibouklis J, Weng T, Zhang B, et al. (2016) Nano carriers for drug transport across the blood-brain barrier. J Drug Target. [Crossref]

70. Aryani A, Denecke B (2016) Exosomes as a Nanodelivery System: a Key to the Future of Neuromedicine? Mol Neurobiol 53: 818-834. [Crossref]

71. Qin Y, Sun R, Wu C, Wang L, Zhang C (2016) Exosome: A Novel Approach to Stimulate Bone Regeneration through Regulation of Osteogenesis and Angiogenesis. Int J Mol Sci 17. [Crossref]

72. Viaud S, Théry C, Ploix S, Tursz T, Lapierre V, et al. (2010) Dendritic cell-derived exosomes for cancer immunotherapy: what's next? Cancer Res 70: 1281-1285. [Crossref]

73. Batrakova EV, Kim MS (2016) Development and regulation of exosome-based therapy products. Wiley Interdiscip Rev Nanomed Nanobiotechnol 8: 744-757. [Crossref]

74. Kim MS, Haney MJ, Zhao Y, Mahajan V, Deygen I, et al. (2016) Development of exosome-encapsulated paclitaxel to overcome MDR in cancer cells. Nanomedicine 12 : 655-664. [Crossref]

75. György B, Hung ME, Breakefield XO, Leonard JN (2015) Therapeutic applications of extracellular vesicles: clinical promise and open questions. Annu Rev Pharmacol Toxicol 55: 439-464. [Crossref]

76. Eitan E, Suire C, Zhang S, Mattson MP (2016) Impact of lysosome status on extracellular vesicle content and release. Ageing Res Rev. [Crossref]

Copyright: $@ 2016$ McKinney CE. This is an open-access article distributed under the terms of the Creative Commons Attribution License, which permits unrestricted use, distribution, and reproduction in any medium, provided the original author and source are credited. 